\title{
Giant Extraarticular Pigmented Villonodular Tenosynovitis; TWIST-MRA Findings
}

\author{
Sıla Ulus ${ }^{1}$, Ercan Karaarslan'1, Harzem Özger², Buğra Alpan³, Bilge Bilgiç
}

${ }^{1}$ Department of Radiology, Acıbadem University School of Medicine, Istanbul, Turkey

${ }^{2}$ Department of Orthopaedics and Traumatology, Istanbul University Istanbul Faculty of Medicine, Istanbul, Turkey

${ }^{3}$ Department of Orthopaedics and Traumatology, JFK Hospital, Istanbul, Turkey

${ }^{4}$ Department of Pathology, Istanbul University Istanbul Faculty of Medicine, Istanbul, Turkey

\section{Introduction}

Pigmented villonodular synovitis (PVNS) is a rare, benign, proliferating disease affecting the synovium of joints, bursae, and tendon sheaths (1). Extra-articular presentation is uncommon. Our purpose is to present magnetic resonance angiography (MRA) findings of pigmented villonodular tenosynovitis (PVNTS) which, to our knowledge, has not been reported previously.

\section{Case Report}

A 32-year-old male presented to the orthopaedics department with pain and swelling in his left ankle. The patient was referred to the radiology department following physical examination. Magnetic resonance imaging (MRI) revealed a $3.5 \times 4.5 \mathrm{~cm}$ lobulated soft-tissue mass surrounding the flexor hallucis longus. The craniocaudal length of the mass in the distal crural and plantar levels was $13 \mathrm{~cm}$ and
$8 \mathrm{~cm}$, respectively. The tendon was intact with no internal pathological signs. Intravenous (IV) contrast was administrated, and time-resolved multiphase four-dimensional MRA (TWIST-MRA) was performed. The mass was hypointense in T1-W (Figure 1a), and heterogeneous iso-mild hyperintense in T2-W images. Punctate and linear hyperintense areas consistent with fatty components and diffuse hypointense signals compatible with haemosiderin pigments were seen in the mass, best visualised in the gradient-echo images (Figure $1 \mathrm{~b}$ ). Post-contrast images showed diffuse homogenous enhancement (Figure 1c). As a conspicuous finding, diffuse and early intense enhancement at the same phase as arteries was noted in the TWIST-MRA images (Figure 2). The integrity of the adjacent tibial cortex was intact. PVNTS was considered in the light of these findings. Before the operation, tru-cut biopsy was performed and the pathology was in concordance with extra-articular PVNS. The mass was completely removed by surgery. Microscopic examination with haematoxylin-eosin staining demonstrated haemosiderin

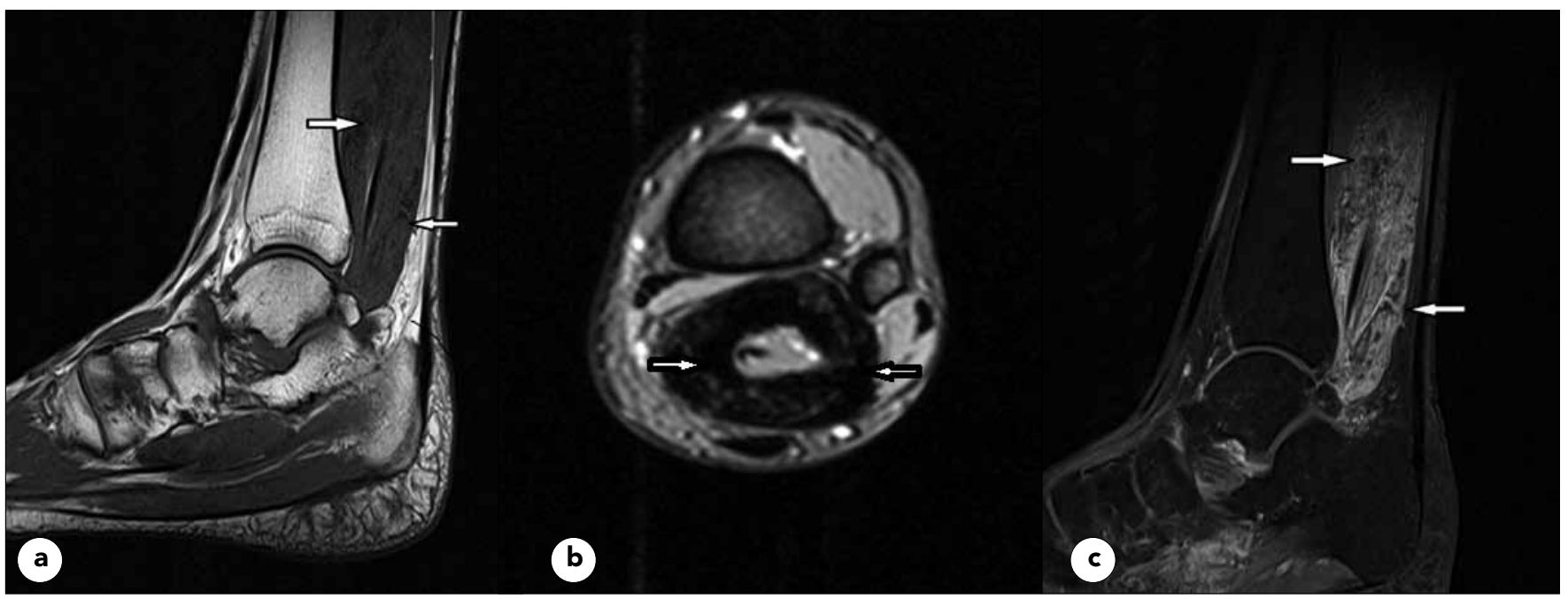

Figure 1. MRI with (a) sagittal T1-W, (b) transverse gradient-echo, and (c) contrast-enhanced T1-W images reveal a mass lesion at the left ankle and distal cruris surrounding the flexor hallucis longus tendon sheath (arrows). The diffuse hypointense areas in the gradient-echo image represent the haemosiderin component. There is diffuse and intense enhancement of the mass in the post-contrast images 


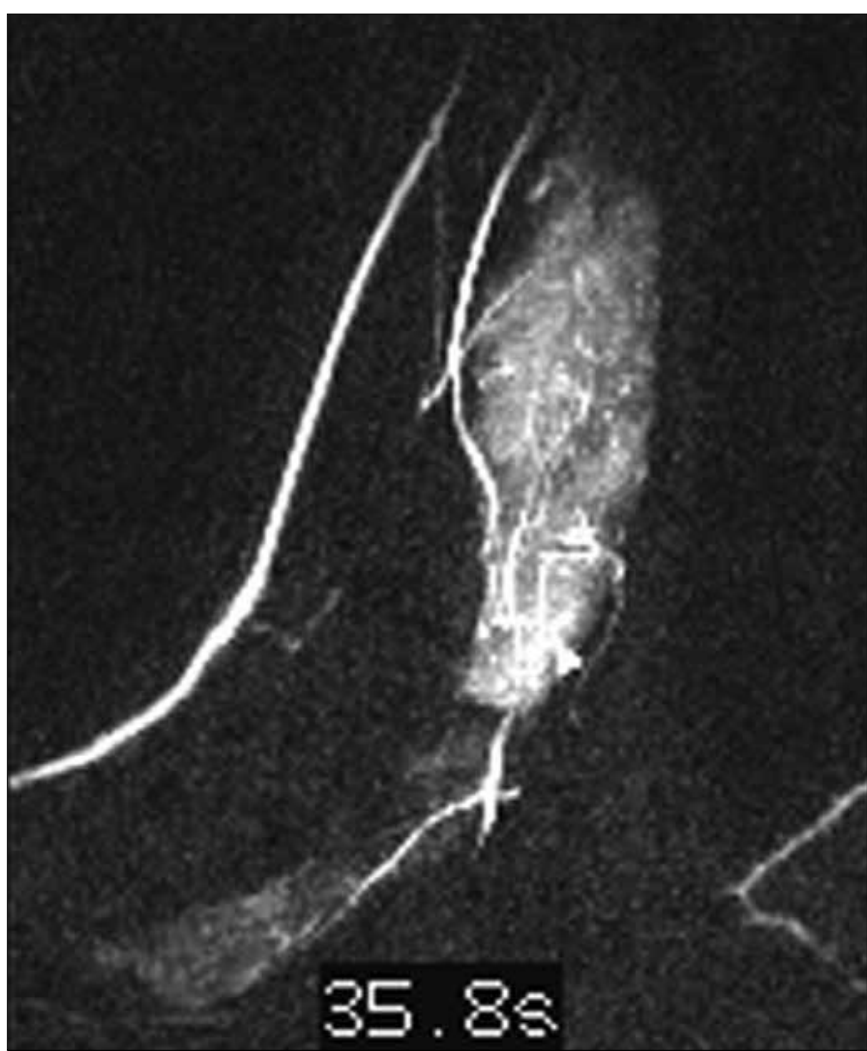

Figure 2. Multiphase four-dimensional TWIST-MRA showing diffuse and intense enhancement of the mass lesion at $\mathbf{3 5 . 8}$ seconds, at the same time as arteries

pigmentation in villous projections of synovial tissue (Figure 3a), tumour cells, multinucleated osteoclasts, and haemosiderin-laden macrophages (Figure 3b), the typical morphology of PVNS. Follow-up MRI performed after 4 months revealed no residue or recurrent tumour. PVNTS showed diffuse and intense enhancement in the early phase of TWIST-MRA. This may be of use as a supplementary marker to gradient-echo images for the diagnosis of PVNTS $(1,2)$.

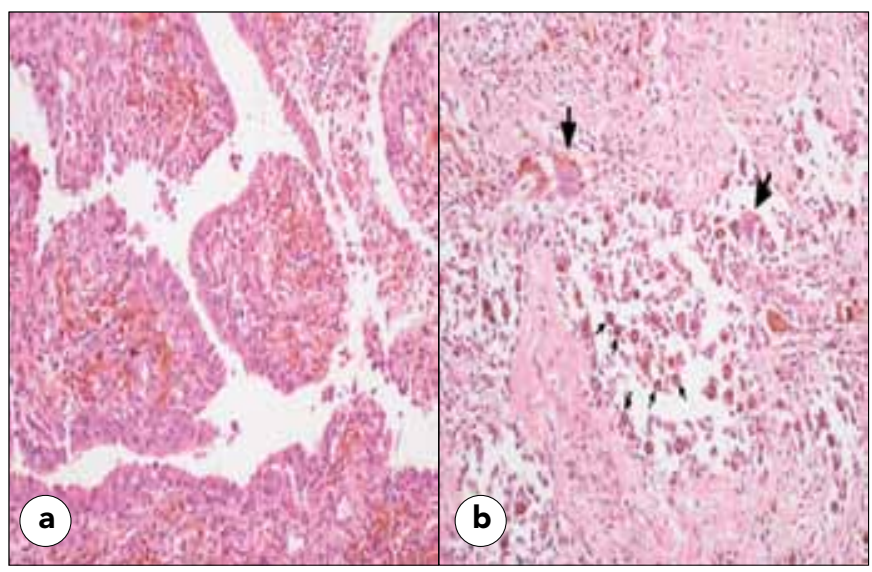

Figure 3. a) Micrograph showing haemosiderin pigmentation in villous projections of synovial tissue. b) Tumour cells, multinucleated osteoclasts (large arrows), and haemosiderin-laden macrophages (small arrows) can be observed in this micrograph

Ethics Committee Approval: N/A.

Informed Consent: Written informed consent was obtained from patients.

Peer-review: Externally peer-reviewed.

Author contributions: Concept - S.U., E.K., H.O.; Design - S.U., E.K.; Supervision - H.U., E.K., B.B.; Resource - E.K., H.U., B.A.; Materials - E.K., H.O.; Data Collection\&/or Processing - S.U., B.A., E.K.; Analysis\&/or Interpretation - E.K., S.U., B.B.; Literature Search - S.U., E.K., B.A.; Writing - S.U., E.K.; Critical Reviews - E.K., H.U.

Conflict of Interest: No conflict of interest was declared by the authors.

Financial Disclosure: No financial disclosure was declared by the authors.

\section{References}

1. Murphey MD, Rhee JH, Lewis RB, Fanburg-Smith JC, Flemming DJ, Walker EA. Pigmented villonodular synovitis:radiologic-pathologic correlation. Radiographics 2008;28:1493-518. [CrossRef]

2. Lin J, Jacobson JA, Jamadar DA, Ellis JH. Pigmented villonodular synovitis and related lesions:the spectrum of imaging findings. AJR Am J Roentgenol 1999;172:191-7. [CrossRef] 